\title{
28 Research Square \\ Prokaryotic Argonaute From Archaeoglobus Fulgidus Interacts With DNA as a Homodimer
}

\section{Edvardas Golovinas}

Vilnius University

\section{Danielis Rutkauskas}

Center for Physical Sciences and Technology

\section{Elena Manakova}

Vilnius University

\section{Marija Jankunec}

Vilnius University

\section{Arunas Silanskas}

Vilnius University

Giedrius Sasnauskas

Vilnius University

Mindaugas Zaremba ( $\sim$ zare@ibt.It)

Institute of Biotechnology, Life Sciences Center, Vilnius University, Sauletekio av. 7, LT-10257, Vilnius, Lithuania.

\section{Research Article}

Keywords: Protein-DNA interactions, Argonaute, pAgo, AFM, smFRET, SAXS, SEC-MALS

Posted Date: December 18th, 2020

DOl: https://doi.org/10.21203/rs.3.rs-128394/v1

License: (c) (i) This work is licensed under a Creative Commons Attribution 4.0 International License. Read Full License

Version of Record: A version of this preprint was published at Scientific Reports on February 25th, 2021. See the published version at https://doi.org/10.1038/s41598-021-83889-4. 


\section{Abstract}

Argonaute (Ago) proteins are found in all three domains of life. The best characterized group is eukaryotic Argonautes (eAgos), which are the core of RNA interference. The best understood prokaryotic Ago (pAgo) proteins are full-length pAgos. They are composed of four major structural/functional domains (N, PAZ, MID and PIWI) and thereby closely resemble eAgos. It was demonstrated that full-length pAgos function as prokaryotic antiviral systems, with the PIWI domain performing cleavage of invading nucleic acids. However, the majority of identified pAgos are shorter and catalytically inactive (encode just MID and inactive PIWI domains), thus their action mechanism and function remain unknown. In this work we focus on AfAgo, a short pAgo protein encoded by an archaeon Archaeoglobus fulgidus. We find that in all previously solved AfAgo structures, its two monomers form substantial dimerization interfaces involving the C-terminal $\beta$-sheets. Led by this finding, we have employed various biochemical and biophysical assays, including SEC-MALS, SAXS, single-molecule FRET and AFM, to show that AfAgo is indeed a homodimer in solution, which is capable of simultaneous interaction with two DNA molecules. This finding underscores the diversity of prokaryotic Agos and broadens the range of currently known Argonaute-nucleic acid interaction mechanisms.

\section{Introduction}

Argonaute (Ago) proteins are found in all three domains of life (bacteria, archaea, and eukaryotes). The best characterized group is eukaryotic Ago (eAgo) proteins. Being the functional core of RNA interference machinery, eAgos are involved in regulation of gene expression, silencing of mobile genome elements, and defense against viruses. From the structural and mechanistic point of view, all eAgos are very similar, as they all use small RNA molecules as guides for sequence-specific recognition of RNA targets, and are monomeric proteins sharing four conserved functional domains, which are organized in a bilobed structure [1]. The $\mathrm{N}$-terminal lobe consists of the $\mathrm{N}$-domain that separates guide and target strands [2], and the PAZ domain responsible for binding the 3 '-terminus of guide RNA; the C-terminal lobe consists of the MID domain, which binds the 5'-terminus of guide RNA, and the PIWI domain, an RNase. Upon recognition of the RNA target, eAgos may either cleave it employing the catalytic activity of the PIWI domain, or, especially eAgo proteins that encode catalytically inactive PIWI domains, recruit partner proteins leading to degradation of the target RNA or repression of its translation [3].

Ago proteins are also identified in $9 \%$ of sequenced bacterial and $32 \%$ archaeal genomes $[4,5]$. Unlike eAgos, which exclusively use RNA guides for recognition of RNA targets, different pAgos may use either RNA or DNA guides and/or targets [6], and may also differ in their structural organization. The best understood prokaryotic Ago (pAgo) proteins are the so called full-length pAgos, which are composed of $\mathrm{N}$, PAZ, MID and PIWI domains, and thus closely resemble eAgo proteins. It has been demonstrated that fulllength pAgos function as prokaryotic antiviral systems, with the PIWI domain performing cleavage of invading nucleic acids [7]. Furthermore, a function beyond immunity - decatenation of circular chromosomes during replication - has also been documented [8]. However, the majority ( 60\%) of identified pAgos are shorter (encode just MID and PIWI domains) and are catalytically inactive due to 
mutations in the PIWI domain. Though similar artificial truncations of eukaryotic Agos preserve most of functionality characteristic to full-length proteins [9-12], the function and mechanism of the naturallyoccurring short catalytically inactive pAgos remain unknown $[4,5]$.

In this work we focus on the short prokaryotic Argonaute AfAgo encoded by a hyperthermophilic archaeon Archaeoglobus fulgidus [4,5] (UniProtKB 028951). Like other short pAgos, AfAgo contains a MID and a catalytically inactive PIWI domains (albeit sequence analysis suggests that AfAgo MID and PIWI domains are closer to those found in full-length, rather than most short pAgos [4,5]). For over a decade it served as a model system for structural and mechanistic studies of Argonaute-nucleic acids interactions [12-14]. It is also one of the first and one of the best structurally characterized prokaryotic Argonautes, with an apo- and 3 dsDNA/RNA-bound structures currently available [15-18]. However, its biological role, in part due to lack of catalytic activity, remains elusive. Inspection of AfAgo structures revealed that regardless of the crystal form and symmetry, two AfAgo subunits in all cases form substantial dimerization interfaces involving the $\mathrm{N}$-terminal residues and/or $\beta$-strands located close to the C-termini. Using various biochemical and biophysical assays, including size exclusion chromatography multi-angle light scattering (SEC-MALS), single-molecule FRET, small angle X-ray scattering (SAXS), and atomic force microscopy (AFM), we show that AfAgo indeed forms a homodimer in solution, and is capable of simultaneous interaction with two DNA molecules. This broadens the range of currently known interaction mechanisms involving nucleic acids and Argonaute proteins.

\section{Materials And Methods}

\section{Protein expression}

The gene encoding WT AfAgo was amplified from Archaeoglobus fulgidus genomic DNA by PCR and cloned into a pETDuet vector, yielding a construct with an $\mathrm{N}$-terminal (His) ${ }_{6}$ tag ( $\mathrm{N}$-terminal protein sequence MGSSHHHHHHSQDP (1.63 kDa) followed by 1-427 aa of WT AfAgo sequence). The deletion in the dimerization mutant AfAgo $\triangle$ was introduced via overlap extension PCR by using two primer pairs, MZ-385/MZ-875 and MZ-383/MZ-876 (Supplementary table S1) for the N- and C-terminal fragments flanking the region to be deleted, respectively. The two PCR products, possessing a 49 bp overlap, were then used as a template for subsequent PCR with the MZ-383/MZ-385 primers, yielding the full-length fragment, which was then cloned into a pETDuet vector. Both proteins were expressed in E. coli strain BL21(DE3). Cells were grown in LB broth in the presence of ampicillin at $37^{\circ} \mathrm{C}$. When A600 of the cell culture reached 0.5 , the incubation temperature was lowered to $16^{\circ} \mathrm{C}, 0.1 \mathrm{mM} \mathrm{IPTG}$ were added, cells incubated for approx. 16 hours at $16^{\circ} \mathrm{C}$ and harvested by centrifugation.

\section{Protein purification}

Harvested cells expressing (His) ${ }_{6}$-tagged WT AfAgo or the dimerization mutant AfAgo $\Delta$ were disrupted by sonication in buffer $\mathrm{A}\left(20 \mathrm{mM}\right.$ Tris $-\mathrm{HCl}\left(\mathrm{pH} 8.0\right.$ at $\left.25^{\circ} \mathrm{C}\right), 500 \mathrm{mM} \mathrm{mM} \mathrm{NaCl}, 5 \mathrm{mM}$ mercaptoethanol) with $2 \mathrm{mM}$ PMSF (phenylmethylsulfonyl fluoride), incubated for $20 \mathrm{~min}$ at $50^{\circ} \mathrm{C}$ and cell debris was 
removed by centrifugation at $48,400 \times \mathrm{g}$ for 1 hour. The supernatant was loaded onto a HiTrap chelating HP column charged with $\mathrm{Ni}^{2+}$ (GE Healthcare) and eluted with a linear gradient (15-500 mM) of imidazole in buffer $\mathrm{A}$. The fractions containing protein were pooled, diluted to $0.2 \mathrm{M}$ of $\mathrm{NaCl}$ with a buffer containing $20 \mathrm{mM}$ Tris- $\mathrm{HCl}\left(\mathrm{pH} 8.0\right.$ at $\left.25^{\circ} \mathrm{C}\right), 10 \%$ (v/v) glycerol, $5 \mathrm{mM}$ 2-mercaptoethanol and incubated for $1 \mathrm{~h}$ at $37^{\circ} \mathrm{C}$ with $1 \mathrm{mM}$ EDTA (ethylenediaminetetraacetic acid) and RNase A/T1 (ThermoFisher Scientific) (1:100). Next, the protein solution was centrifuged at $48,400 \mathrm{xg}$ for 30 minutes, the supernatant containing RNA-free AfAgo was loaded onto a HiTrap Heparin HP column (GE Healthcare), and eluted using a $0.2-1.0 \mathrm{M} \mathrm{NaCl}$ gradient. Finally, the protein was run through the HiLoad 16/600 Superdex 200 column (GE Healthcare) in buffer A and dialyzed against $20 \mathrm{mM}$ Tris- $\mathrm{HCl}\left(\mathrm{pH} 8.0\right.$ at $25^{\circ} \mathrm{C}$ ), $500 \mathrm{mM} \mathrm{NaCl}, 50 \%$ (v/v) glycerol.

\section{SEC-MALS}

The samples of WT AfAgo or AfAgo $\triangle$ in the absence of nucleic acids (final protein concentration $1.0 \mathrm{mg} / \mathrm{mL}$ or $20 \mu \mathrm{M}$ in terms of monomer, injected volume $2.0 \mathrm{~mL}$ ) were separated using a HiLoad $16 / 60$ Superdex 200 prep grade column (GE Healthcare). The column was equilibrated with a buffer containing $100 \mathrm{mM}$ Tris- $\mathrm{HCl} \mathrm{pH} \mathrm{8.0,1000} \mathrm{mM} \mathrm{NaCl}$ and $0.04 \%(\mathrm{w} / \mathrm{v}) \mathrm{NaN}_{3}$, the flow rate was $0.5 \mathrm{~mL} / \mathrm{min}$. Samples of AfAgo with 5'-phosphorylated MZ-1289 DNA (final concentration $5 \mu \mathrm{M}$ protein monomer and $5 \mu \mathrm{M}$ DNA oligoduplex) were separated on a Superdex 200 Increase 10/300 GL column equilibrated with a buffer containing $15 \mathrm{mM}$ Tris- $\mathrm{HCl} \mathrm{pH} \mathrm{8.0,150} \mathrm{mM} \mathrm{NaCl}, 5 \mathrm{mM} \mathrm{MgCl}_{2}, 0.5 \mathrm{mM}$ 1,4-dithiothreitol and $0.04 \%(\mathrm{w} / \mathrm{v}) \mathrm{NaN}_{3}$, flow rate was $0.4 \mathrm{~mL} / \mathrm{min}$. The light scattering signals were monitored on a miniDawn TREOS II detector, concentrations of apo-protein samples were measured using an Optilab T-rEX refractive index detector (Wyatt Technologies), concentrations of protein-DNA complexes were measured using both refractive index and UV absorption (Waters 2487 UV detector) readings. Data were analyzed in Astra software (Wyatt Technologies) using dn/dc values of $0.185 \mathrm{~g} / \mathrm{mL}$ and $0.170 \mathrm{~g} / \mathrm{mL}$ for protein and DNA, respectively. Scattering data of protein-DNA complexes was analyzed using the "protein conjugate" method in Astra; the required DNA and apo-protein UV extinction coefficients were determined experimentally using the "UV extinction from RI peak" method and the DNA-only and apo-protein samples.

\section{Small angle X-ray scattering}

Small angle scattering data of WT AfAgo and monomeric mutant were collected at the P12 EMBL beamline on the PETRA III ring of the DESY synchrotron in Hamburg, Germany [19]. Details of data collection and principal structural parameters are presented in Supplementary table S2 and Supplementary Fig. S1. Protein complexes with DNA (MZ-1289) were transferred to sample buffer (20 mM Tris- $\mathrm{HCl}\left(\mathrm{pH} 7.5\right.$ at $25^{\circ} \mathrm{C}$ ), $5 \mathrm{mM} \mathrm{MgCl}_{2}, 150 \mathrm{mM} \mathrm{NaCl}$ and $2 \mathrm{mM}$ 1,4-dithiothreitol) using Illustra NAP columns (GE Healthcare). Apo AfAgo sample was measured in the same buffer containing $500 \mathrm{mM}$ $\mathrm{NaCl}$. 
Dimeric AfAgo complex with MZ-1289 was analyzed by SEC-SAXS with FPLC (Agilent) using WyattMALS-DLS detection system. The AfAgo+MZ-1289 was concentrated to $175 \mu \mathrm{M}$ and loaded on the column Superdex 200 Increase 10/300 (GE Healthcare) equilibrated with the sample buffer. Frames collected during the complete SEC run (flow rate $0.5 \mathrm{ml} / \mathrm{min}, 3000$ frames) were analyzed with CHROMIXS [20], frames corresponding to the peak were averaged and processed. Ab initio shape determination was carried out by generating 20 independent DAMMIF [21] models using parameterized scattering curves created by GNOM [22] under P2 symmetry restraints. Models were clustered by DAMCLUST [23] and models forming a cluster were averaged by DAMAVER [24] and used as a starting model for an additional run of DAMMIN [25].

SAXS measurements performed with a range of AfAgo $\Delta$ concentrations $(1-10 \mathrm{mg} / \mathrm{ml}$, both the apo-form and a complex with MZ-1289 DNA), showed significant protein aggregation, particularly pronounced with the apo-AfAgo $\Delta$. The pseudo-chain dummy residues models of the complex generated by GASBOR [26] were superimposed with crystallographic dimers of AfAgo as well as with the monomeric AfAgo-DNA complex using SUPCOMB [27] applying step-wise shift (5 $\AA$ ) along the principal axis of the model as described in [28].

Comparison of the SAXS data with crystal structures was carried out by CRYSOL [29] (Fig. 1). Particle volume and $\mathrm{M}_{\mathrm{w}}$ estimations were performed using several methods (Supplementary table S3 and references therein).

\section{DNA fragments}

DNA fragments were assembled and prepared as depicted in Supplementary Fig. S2. All full-length DNA fragments were subsequently purified from an agarose gel using a runVIEW system (Cleaver Scientific, UK), precipitated with sodium acetate/isopropanol, washed with $75 \%(\mathrm{v} / \mathrm{v})$ ethanol and resuspended in water.

\section{AFM sample preparation and imaging}

DNA-protein complexes were formed by incubating the DNA fragment ( $5 \mathrm{nM})$ with WT AfAgo or AfAgo $\triangle$ (concentration in terms of monomer $50 \mathrm{nM}$ ) for $5 \mathrm{~min}$ at room temperature in the Binding Buffer HEPES (33 mM HEPES (pH 7.8 at $\left.25^{\circ} \mathrm{C}\right), 66 \mathrm{mM} \mathrm{CH}_{3} \mathrm{COOK}, 5 \mathrm{mM}\left(\mathrm{CH}_{3} \mathrm{COO}\right)_{2} \mathrm{Mg}$,) in a total volume of $50 \mu \mathrm{l}$. Next, the protein-DNA complexes were cross-linked with $2.5 \%(\mathrm{w} / \mathrm{v})$ glutaraldehyde for $20 \mathrm{~min}$. Glutaraldehyde was then quenched with an excess of the Tris buffer $(33 \mathrm{mM}$ Tris-acetate $(\mathrm{pH} 7.8$ at $\left.25^{\circ} \mathrm{C}\right), 66 \mathrm{mM} \mathrm{CH}_{3} \mathrm{COOK}, 5 \mathrm{mM}\left(\mathrm{CH}_{3} \mathrm{COO}\right)_{2} \mathrm{Mg}$,). The resultant reaction solution after 10 -fold dilution with Tris buffer was deposited onto modified mica at room temperature as described below.

Freshly cleaved muscovite mica (grade IV, SPI supplies Inc., USA) was incubated in a mixture of 1-(3aminopropyl)-silatrane (APS) solution for $30 \mathrm{~min}$ to prepare functionalized APS-mica, as described previously for the preparation of protein-DNA complexes [30]. $50 \mu$ of DNA-protein complex solution was deposited on APS-mica for 5 min. After incubation the mica surface was immersed into deionized water 
for $5 \mathrm{~min}$, flushed with excess water and then dried under a flow of nitrogen. The images were acquired in the air with "Dimensionlcon" (Bruker, Santa Barbara, CA) microscope system in tapping mode. Probes with nominal spring constants of $\sim 5$ or $40 \mathrm{~N} / \mathrm{m}$ were used. Typically, the images were collected at a speed of $0.6 \mathrm{~Hz}$ and a resolution of $1024 \times 1024$ pixels, scan size $2 \mu \mathrm{m} \times 2 \mu \mathrm{m}$.

\section{Single-molecule fluorescence microscopy}

The overall idea of fluorescence burst data acquisition of single diffusing molecules in alternating laser excitation (ALEX) mode was based on [31]. The principal opto-mechanical layout of the experiment is shown in Supplementary Fig. S3 and described in Supplementary Data.

The measurement of single surface-immobilized molecules with the excitation in the total internal reflection mode (TIR) was performed on the same setup exploiting its alternative functionality as described previously [32]. Briefly, the objective was 100× 1.4 Oil Plan Apo VC (Nikon), the fluorescence signal was split by T640lpxr-UF2 dichroic mirror (Chroma) and the different spectral channels were projected on the same EMCCD (DU-897ECS0-UVB, Andor).

\section{Sample cell preparation for single molecule measurements}

FRET bursts measurements were performed in a chambered coverglass well (155411, Nunc Lab-Tek, Thermo Scientific). The reaction volume was $200 \mu \mathrm{l}$. The reaction buffer (RB) was $33 \mathrm{mM}$ Tris-acetate (pH 7.9 at $\left.20{ }^{\circ} \mathrm{C}\right), 66 \mathrm{mM} \mathrm{CH}_{3} \mathrm{COOK}, 5 \mathrm{mM}\left(\mathrm{CH}_{3} \mathrm{COO}\right){ }_{2} \mathrm{Mg}$, and $0.1 \mathrm{mg} / \mathrm{ml} \mathrm{BSA}$ (bovine serum albumin).

The DNA concentration was 17-50 pM. Measurements at different protein concentrations were carried out by adding to the reaction small volumes of protein diluted in RB in "Protein LoBind" $1.5 \mathrm{~mL}$ tubes (Eppendorf). No oxygen-scavenging or triplet-quenching additives were used.

Measurements of surface-immobilized DNA fragments were performed in a flow cell assembled from a six-channel Sticky-Slide VI 0.4 (Ibidi) and a coverslip functionalized with PEG (polyethylene glycol) derivatives as described in detail in [32]. The flow cell was incubated with $5 \mu \mathrm{g} / \mathrm{ml}$ of Neutravidin (Molecular probes) in RB for 2 min, washed with RB, incubated with 5 pM DNA in RB until the density of the surface-immobilized DNA fragments appeared to be appropriate, and washed with RB. For the measurement, $10 \mathrm{nM}$ solution of AfAgo in RB supplemented with 1\% (w/v) glucose (TCl Europe), $2.5 \mathrm{mM}$ Trolox (Sigma-Aldrich), and $15 \mathrm{U} / \mathrm{ml}$ glucose oxidase (Sigma-Aldrich) was injected into the cell. Trolox was treated with UV light for 20 min according to Cordes et al. [33]. Single molecule data analysis was performed as described in Supplementary Data.

\section{Results}

\section{AfAgo is a homodimer in the available X-ray structures}

AfAgo is a 427 amino acid (aa) $49.2 \mathrm{kDa}$ prokaryotic Argonaute protein found in the hyperthermophilic archaeon Archaeoglobus fulgidus. To date, four AfAgo structures, both of the apo-form and bound to 
DNA and RNA duplexes were solved [15-18]. AfAgo monomer is composed of two major domains, the Nterminal MID (residues 38-167), and the C-terminal PIWI (residues 168-427) [15]. The MID domain specifically binds the 5 '-phosphorylated end of the presumed guide DNA/RNA strand, and also makes contacts to the complementary target DNA/RNA strand [16-18]. The PIWI domain makes contacts to both guide and target DNA/RNA strands, but is catalytically inactive due to mutations in the RNase H-like catalytic center. Inspection of available AfAgo structures (Supplementary table S4) revealed that in all structures known so far, AfAgo subunits form substantial homodimerization interfaces, which were not further scrutinized by authors of the corresponding structural studies. The dimerization interface in the AfAgo-dsRNA structure (PDB ID 1ytu) is asymmetric, and primarily involves the $C$-terminal $\beta$-strands (residues 296-303) from both subunits present in the asymmetric unit that together form a parallel $\beta$ sheet, and the $\mathrm{N}$-terminal residues from one of the subunits (Fig. 1a). The dimer formed in this case is compact (henceforth, a "closed" dimer). In contrast, dimerization interfaces in three other cases (PDB IDs $1 \mathrm{w} 9 \mathrm{~h}, 2 \mathrm{bgg}$ and $2 \mathrm{w} 42$ ) are nearly symmetrical with respect to the secondary structure elements involved (albeit in PDB IDs $2 \mathrm{bgg}$ and $2 \mathrm{w} 42$ they belong to different protein chains present in the asymmetric unit): the $C$-terminal $\beta$-strands form 8-strand $\beta$-barrels, with the sheets from different subunits interacting via strands $\beta 14$ (residues 297-302) and $\beta 15$ (residues 314-318, Fig. 1b). The resultant dimers are less compact (henceforth, "open" dimers).

The solvent accessible surface areas buried at the dimerization interfaces in both "open" and "closed" dimers are classified as "significant" by the PISA server (https://www.ebi.ac.uk/pdbe/pisa/pistart.html, [35]). This observation prompted us to test the oligomeric state, the possible dimerization mode, and mechanism of nucleic acid binding of AfAgo in solution using various biochemical and biophysical techniques. For that purpose, we used two variants of AfAgo: the full-length wild-type protein (henceforth, WT AfAgo), and a dimerization mutant AfAgo lacking the 296-303 amino acid residues responsible for the majority of dimerization contacts in both the "closed" and "open" homodimers (henceforth, AfAgo $\Delta$, Fig. 1a-b). Both proteins were successfully purified as described in Materials and Methods.

\section{SEC-MALS analysis}

First, we tested the oligomeric state of WT AfAgo and AfAgo $\Delta$ proteins using size exclusion chromatography - multi angle light scattering (SEC-MALS). We find that WT AfAgo elutes from the SEC column as a polydisperse peak (Fig. 2a), with the $M_{w}$ values ranging from $91.7 \mathrm{kDa}$ at the left-hand side of the peak (close to the theoretical $M_{w}$ of WT AfAgo homodimer, $101.6 \mathrm{kDa}$ ) to $59.5 \mathrm{kDa}$ at the righthand side (still considerably higher than $\mathrm{M}_{\mathrm{w}}$ of a WT AfAgo monomer, $50.8 \mathrm{kDa}$ ). AfAgo $\triangle$ formed a far broader irregular peak, covering $\mathrm{M}_{\mathrm{w}}$ values from $87 \mathrm{kDa}$ (close to $\mathrm{M}_{\mathrm{w}}$ of AfAgo $\Delta$ dimer, $99.8 \mathrm{kDa}$ ) to approx. $49 \mathrm{kDa}$ (close to AfAgo $\triangle$ monomer, $49.9 \mathrm{kDa}$ ). We conclude that WT AfAgo indeed forms homodimers, which under our experimental conditions ( $\sim 10 \mu \mathrm{M}$ concentration in the sample, $\sim 1.5 \mu \mathrm{M}$ concentration on the column) are relatively unstable and partially dissociate into monomers. Deletion of the $\mathrm{C}$-terminal residues in the AfAgo $\Delta$ protein further decreased the stability of the dimer, in line with their proposed role in dimerization (Fig. 1). Intriguingly, the differences between WT AfAgo and AfAgo $\triangle$ oligomeric states were more pronounced in their DNA-bound forms (AfAgo-DNA and AfAgo $\triangle$-DNA, 
respectively): the majority of WT AfAgo-DNA eluted as a 2:2 protein:DNA complex, and the major peak of AfAgo $\triangle$-DNA matched a 1:1 protein:DNA complex (Fig. 2b).

\section{SAXS measurements}

To characterize the conformation of AfAgo in solution, we have performed small angle X-ray scattering (SAXS) measurements using WT AfAgo-DNA and AfAgo $\triangle$-DNA complexes. Two types of data analysis were performed: (i) the ab initio shapes of the complexes in solution were calculated and superimposed with the X-ray AfAgo structures, and (ii) the theoretical scattering data was calculated for the crystallized DNA-bound AfAgo monomer, "open" (PDB ID: 2w42 and 1w9h) and "closed" (PDB ID: 1ytu) dimers, and compared to experimental SAXS scattering data of AfAgo-DNA and AfAgo $\triangle$-DNA (Fig. 2). The "closed" AfAgo dimer fits the AfAgo-DNA SAXS data better than the "open" dimer, as judged from the real space fit

and the $\chi^{2}$ (Fig. 2c) parameters that reflect the agreement between scattering functions of corresponding crystal structures and SAXS experiments (Fig. 2c), implying that in solution WT AfAgo predominantly forms a "closed" dimer. As expected, AfAgo monomer gave the best fit to the AfAgo $\triangle$-DNA SAXS data (Fig. 2c, right column). The SAXS molecular weights calculated for WT AfAgo-DNA (between 94.2 and $106.9 \mathrm{kDa}$, Supplementary table S3) agreed with the expected mass of the dimer complexed with dsDNA (119 kDa). The SAXS $M_{w}$ for the AfAgo $\triangle$-DNA (between 55.4 and $67.9 \mathrm{kDa}$ ) confirmed its monomeric state.

\section{Direct visualization of AfAgo-induced DNA loops by AFM}

We have observed the dimeric state of WT AfAgo in X-ray structures, SEC-MALS and SAXS measurements, i.e. techniques that all require relatively high (micromolar and higher) protein and DNA concentrations. This raises a question if AfAgo dimerisation and the ability to simultaneously interact with two nucleic acid molecules are relevant in solution at far lower protein and DNA concentrations. To address this question we have examined AfAgo interactions with long DNA molecules by AFM and single molecule FRET.

For direct visualization of protein-DNA complexes, AfAgo and DNA were deposited on APS-mica and imaged using tapping AFM. A typical AFM image of AfAgo-DNA complexes is shown in Fig. 3. Several types of protein-DNA complexes, shown as enlarged insets in Fig. 3, were observed: (i) linear DNA with a protein molecule bound to one DNA end; (ii) linear DNA with protein molecules bound to both DNA ends; (iii) ring-shaped (looped) DNA. Other species, including naked DNA, or more complex structures, involving, e.g., protein bound to two DNA fragments, were also observed, but were not quantified. We find that the relative distribution of different complexes varied dramatically for WT AfAgo and the dimerization mutant AfAgo $\triangle$ (Table 1). The ring-shaped DNA-protein complexes are the dominant species observed with WT AfAgo ( $55 \%$ or 114 out of 208 complexes). The minor fraction of DNA molecules had either protein bound to one end $(29 \%, 61$ out of 208 complexes) or to both ends (16\%, 33 out of 208). In the case of AfAgo $\Delta$, the majority of complexes had protein bound to both DNA ends ( $59 \%$, or 187 out of 319 , Table 1 ), and a much lesser fraction (12\%, or 38 out of 319$)$ were ring-shaped structures. We assume that ring-shaped 
DNA molecules are formed by dimeric WT AfAgo bound to both termini of the DNA fragment, in a similar manner as observed in the X-ray structures. A prominent decrease in ring-shaped DNA in the AfAgo $\Delta$ samples is consistent with its impaired dimerization. The remaining looped complexes in this case are likely formed due to residual ability of AfAgo $\Delta$ to form dimers, though we cannot exclude inadvertent cross-linking of DNA-bound AfAgo $\Delta$ monomers with glutaraldehyde during sample preparation (see Materials and Methods for details).

\section{WT AfAgo induces DNA loops in solution}

To further characterize AfAgo-DNA interactions at nanomolar concentrations, we have examined AfAgoDNA interactions using single-molecule Förster resonance energy transfer (smFRET). If AfAgo homodimer simultaneously interacts with two ends of the same DNA molecule, the induced DNA loops can be monitored as a change in FRET efficiency between dyes tethered close to DNA ends (Fig. 4a). Utilization of a single dual-labeled DNA substrate (rather than two short DNA duplexes carrying different fluorescent labels) increases the probability of AfAgo interaction with both DNA ends at low reactant concentrations required for the single-molecule setup.

We have designed a 569 bp DNA construct, which was labelled with a pair of FRET fluorophores, Cy3B and Atto647N, each attached to thymine bases 3 nt away from the respective DNA termini via a C6 linker (Supplementary Fig. S2). The positions of FRET labels were selected such that upon binding of both DNA ends by an AfAgo dimer, the distance between the label attachment sites (irrespective of the AfAgo dimerization mode), is favorable for FRET (Supplementary Fig. S4). To promote tighter binding, both DNA ends were phosphorylated, and the 5'-terminal nucleotides were adenines, since AfAgo, like Argonaute CbAgo from Clostridium butyricum [36], has a preference for a 5'-terminal A (publication in preparation).

AfAgo interaction with the DNA fragment was monitored by analyzing the fluorescence bursts of single diffusing DNA fragments (Fig. 4b). As described in Supplementary Materials and Methods, for each DNA molecule we have calculated the stoichiometry parameter S, which is close to 0.5 for DNA molecules labeled with both fluorophores, approx. 0 for the acceptor-only DNA, and close to 1.0 for the donor-only DNA, and the proximity ratio $E$, which is expected to be higher for looped DNA molecules with the FRET dyes brought into close proximity than for unlooped DNA molecules.

The E-S histogram of DNA alone (Fig. 4c, left) exhibits a prominent population with low E and intermediate $S$ values, which corresponds to dual-labeled unlooped (zero-FRET) DNA molecules. The two minor populations observed in the histogram correspond to donor-only (low E/high S) and acceptor-only (high E/low S) DNA fragments.

The E-S histogram of DNA in the presence of WT AfAgo exhibits an additional population (intermediate S and intermediate E, Fig. 4c, right), which presumably represents DNA molecules looped by WT AfAgo. The fraction of looped and unlooped DNA molecules was quantified by fitting a sum of two Gaussian functions to the 2D histogram of E values of dual-labeled molecules (Fig. 4C, right), and finding the areas under the Gaussian with a relatively high E center (representing looped DNA) and a Gaussian with a near- 
zero $\mathrm{E}$ center (representing unlooped DNA). DNA looping efficiency $\mathrm{K}$ was then defined as the ratio of the two areas.

We have measured the ratio $\mathrm{K}$ at different WT AfAgo concentrations (Fig. 4d). It increased monotonously with increasing WT AfAgo concentration until it reached the maximum value of 2.5 (corresponds to about $70 \%$ of looped DNA molecules) at $1 \mathrm{nM} \mathrm{WT}$, but decreased as the protein concentration was increased further.

A similar set of single molecule experiments was performed with the dimerization mutant AfAgo $\triangle$. As shown in Fig. $4 \mathrm{~d}$, the ratio $\mathrm{K}$ at all AfAgo $\triangle$ concentrations tested was close to zero, indicating that AfAgo $\triangle$ was unable to induce DNA loops. Lack of DNA looping was not due to lack of DNA binding, as shown by electrophoretic mobility shift assay (Supplementary Fig. S5). Moreover, AfAgo $\Delta$ competes with WT AfAgo for DNA ends, as the $\mathrm{K}$ value observed in a competition experiment performed with equal concentrations of WT AfAgo dimer and AfAgo $\triangle$ monomer was considerably lower than in an experiment with WT AfAgo alone (Fig. 4d). Taken together, efficient DNA looping observed with WT AfAgo, and impaired looping by the dimerization interface mutant $\operatorname{AfAgo} \Delta$, provide further support for the ability of WT AfAgo dimer to simultaneously bind two DNA ends in solution.

\section{Dynamics of WT AfAgo-induced DNA loops}

To explore the dynamics of the WT AfAgo-induced DNA looping events, we have used total internal reflection fluorescence (TIRF) microscopy to perform single molecule FRET experiments on surfaceimmobilized DNA (Fig. 5). For that purpose we used a DNA fragment that was essentially identical to the one used for single-molecule studies in solution, except that it carried a biotin 386 bp away from the donor end for surface immobilization (Supplementary Fig. S2). After verifying that WT AfAgo induces loops on this substrate in solution (Supplementary Fig. S6b), we immobilized the biotinylated DNA on a surface and then recorded fluorescence movies in the absence or in the presence of WT AfAgo (Supplementary Fig. S6a). From each frame of the movie we then extracted donor and acceptor intensities for individual DNA fragments, selected trajectories with anti-correlated changes of the donor and acceptor intensities (indicating the occurrence of FRET) and calculated the time courses of the proximity ratio, E. An example of such a trajectory is presented in Fig. 5c. In a control with no AfAgo we could find no DNA fragments exhibiting FRET (Fig. 5b).

The single-molecule population and time-averaged $E$ values exhibits two peaks with maxima at 0.09 and 0.36 , corresponding to the unlooped and looped DNA molecules, respectively (Fig. 5d, right). These $\mathrm{E}$ values are also in good agreement with the $E$ values obtained from the measurement in solution (Fig. 4c).

A superficial inspection of E trajectories of individual DNA fragments revealed that their looping dynamics are rather diverse. There exist trajectories with the looped state lasting the whole measurement, whereas other trajectories are more dynamic with a number of transitions between the looped and unlooped states (Fig. 5c, Supplementary Fig. S6c, d). The looped E state also exhibits more subtle 
dynamics (Supplementary Fig. S6d), which could probably be attributed to the conformational flexibility of AfAgo at the dimerization interface.

To quantify the looped state duration we first idealized the $E$ trajectories using HMM with a two-state model in QuB software [37] (Supplementary Materials and methods) (Fig. 5e), and then built the cumulative histogram of the looped state durations (Fig. 5f). The trajectory edge dwells were not omitted in order to preserve the information on the occurrence of states lasting during the whole trajectory. The exponential factor of a single-exponential fit to the cumulative histogram was $33 \pm 1 \mathrm{~s}$. However, the maximum recorded looped state duration is limited by the duration of our measurement (200 s) and the duration of the fluorescent state of the fluorophores before photobleaching. The value of the exponential factor thus sets the lower limit for the looped state duration.

\section{Discussion}

All characterized long Argonaute proteins interact with their RNA and/or DNA targets as monomers, binding a single copy of each guide and target nucleic acids. Surprisingly, we reveal here that AfAgo, a prokaryotic Argonaute from the hyperthermophilic archaeon Archaeoglobus fulgidus, follows a different mechanism, which involves homodimerization and simultaneous interaction with two guide-target nucleic acid duplexes.

First, we show that AfAgo is a homodimer in all previously solved X-ray structures, including apo-protein, and complexes with RNA and DNA (Supplementary table S4). Two types of AfAgo dimerization interfaces formed by the C-terminal $\beta$-sheets are observed in the structures. Both types of interfaces bury a comparable solvent-accessible surface area (Supplementary table S4), but result in distinct arrangement of AfAgo subunits relative to one another, which we term "closed" and "open" dimers (Fig. 1 a and b, respectively). The "closed" type of AfAgo homodimer, formed when the interface involves both the Nterminal residues and the $\mathrm{C}$-terminal $\beta$-strands (Fig. 1a), provides a better fit to our SAXS data, suggesting that it is the major type of DNA-bound WT AfAgo dimer present in solution (Fig. 2c). It remains to be determined if formation of the alternative "open" dimer observed in several structures (Fig. 1b and Supplementary table S4) was influenced by crystal packing, or rather it is an alternative less abundant arrangement of AfAgo subunits that co-exists in solution at equilibrium with the "closed" form. As expected, removal of the $\beta$-strands located at the intersubunit interface (variant AfAgo $\Delta$ ) impaired AfAgo dimerization (Fig. 2).

Simultaneous binding of WT AfAgo homodimer to both ends of a linear DNA fragment, one DNA end per AfAgo monomer, would result in a DNA loop. Formation of such looped DNA molecules upon incubation with WT AfAgo was directly visualized using AFM (Fig. 3). As shown in Table 1, ring-shaped AfAgo-DNA complexes constitute the majority of all protein-DNA complexes detected. Considerable decrease in the fraction of looped DNA complexes in the case of the dimerization interface mutant AfAgo $\triangle$ (Table 1 ) provides further proof that DNA looping is indeed mediated by the dimeric form of AfAgo protein. 
To further characterize the interaction of WT AfAgo with DNA in solution, we have performed singlemolecule FRET measurements (Fig. 4) using a DNA fragment labelled with fluorescent labels close to DNA ends. Design of the DNA substrate ensured that binding of WT AfAgo dimer to both DNA ends would bring the fluorophores into close proximity, resulting in FRET. Comparison of donor/acceptor channel records for free DNA and DNA with either WT AfAgo or dimerization-incapable AfAgo $\triangle$ confirmed that only WT AfAgo efficiently forms DNA loops, yet again implying that DNA looping is mediated by dimeric AfAgo.

Assuming that wt AfAgo in its apo-form is an unstable dimer in solution (Fig. 2a), at least two types of mechanisms can be proposed for the formation of the WT AfAgo dimer / looped DNA complex, one involving apo-AfAgo homodimers (Fig. 6, left), the other involving apo-AfAgo monomers (Fig. 6, right). In the first scenario, the reaction may proceed via (i) association of free DNA (species "0") with a single WT AfAgo dimer, which binds to one DNA end (species "1"); (ii) capture of the second DNA terminus by the DNA-bound AfAgo dimer in an intramolecular reaction, resulting in a looped complex (species "2"); (iii) alternatively, association of the second WT AfAgo dimer with the unoccupied second DNA end of species " 1 " leads to species " 3 ", which is no longer capable of loop formation. Such mechanism was demonstrated for many proteins capable of DNA looping, including restriction endonucleases $[32,38,39]$ and transposases [40-43]. In the alternative scenario, the DNA looping involves (i) binding of a single AfAgo monomer to the first DNA end (species "4", Fig. 6); (ii) binding of the second AfAgo monomer to the second DNA end (species " 5 "); (iii) association of two DNA-bound monomers into the looped complex "2"; (iv) association of additional DNA-unbound AfAgo monomers with DNA-bound AfAgo subunits, process that occludes loop formation (species " 6 " Fig. 6). Both these reaction pathways predict that at elevated protein concentrations the number of looped complexes should decrease, due to simultaneous binding of separate AfAgo dimers to both DNA ends (species " 3 "). Single molecule FRET experiments in solution support this prediction. Indeed, the amount of looped DNA molecules increases until an optimal protein concentration is reached (approx. $1 \mathrm{nM}$ dimer in our experimental setup, Fig. 4d), but declines upon further increase in WT AfAgo concentration. However, the relative contribution of pathways involving WT AfAgo monomers and dimers into the overall DNA looping reaction remains to be established.

Single-molecule measurements on immobilized DNA allowed us to assess the dynamic properties of WT AfAgo-induced DNA loops. We find that (i) the DNA loops induced by WT AfAgo are relatively stable, with the lower limit estimate for the loop duration exceeding $30 \mathrm{~s}$ (Supplementary Fig. S6c); (ii) the proximity ratio $E$ of the looped complexes changes over time, suggesting intrinsic dynamics of the AfAgo dimer, presumably attributable to the flexible dimerization interface (Supplementary Fig. S6d).

\section{Conclusions}

The ability of WT AfAgo to form homodimers and bring two nucleic acid fragments into close proximity, to the best of our knowledge, was not previously described for any Argonaute protein. This finding underscores the diversity of prokaryotic Agos in general and broadens the range of currently known 
Argonaute-nucleic acid interaction mechanisms. In particular, it also raises questions regarding the currently unknown AfAgo function. Simultaneous interaction with two target sites in the case of restriction endonucleases is believed to increase specificity by preventing inadvertent cleavage of lone unmodified target sites [39,44]. However, since AfAgo has no intrinsic nuclease activity, it cannot be directly involved in host defense against invading nucleic acids, as recently proposed for the catalytically active full-length pAgos [45,46]. Instead, the ability of WT AfAgo to form stable synaptic complexes with two DNA ends is reminiscent of transposases [40-43], Cas1-Cas2 integrases [47,48], and (retro)viral integrases [49,50], which often bring the reactive 3'-OH groups of two DNA ends into proximity of the integration site. AfAgo thus could serve as the recognition module for the integrated DNA fragment; target DNA recognition, binding and catalysis of the integration reactions would require involvement of additional, currently unknown, partner proteins. Intriguingly, some pAgos were recently shown to enhance homologous recombination in bacteria, a process that involves direct interaction of a catalytically active or inactive pAgo PIWI domain with RecA recombinase [51]. It remains to be seen if homologous recombination is also enhanced by AfAgo, and if it is affected by AfAgo dimerization. We currently continue structural-functional studies of AfAgo and related short prokaryotic Argonautes, primarily focusing on identification of their partner proteins and possible biological roles.

\section{Declarations}

\section{Acknowledgement}

Authors gratefully acknowledge prof. Virginijus Siksnys for fruitful discussions and comments on the manuscript. Authors also acknowledge Audra Ruksenaite for mass spectrometry of AfAgo proteins, Tomas Urbaitis for generation of the dimerization mutant AfAgo $\Delta$ and initial experiments, and prof. Gintaras Valincius for support. The synchrotron SAXS data was collected at beamline P12 operated by EMBL Hamburg at the PETRA III storage ring (DESY, Hamburg, Germany). We would like to thank Dr. Melissa Graewert for the assistance in using the beamline.

\section{Contributions}

AS purified the target proteins. EM performed SAXS measurements and crystallographic analysis. GS performed SEC-MALS experiments, DR and EG performed smFRET experiments. MJ performed AFM measurements. GS, DR, EM and EG drafted the manuscript. MZ designed and coordinated the study, and critically edited the manuscript. All authors read and approved the final manuscript.

\section{Accession codes}

A. fulgidus (ATCC 49558) AfAgo, UniProtKB entry 028951.

\section{Competing interests}

The authors declare no competing interests. 


\section{Funding}

This work was supported by the Research Council of Lithuania [S-MIP-17-61 to M.Z.]. The access to P12 was supported by the Horizon 2020 programme of the European Union, iNEXT (H2020 Grant \# 653706). Funding for open access charge: the Research Council of Lithuania.

\section{AUTHOR NOTES}

\section{Corresponding Author}

*Mindaugas Zaremba - Institute of Biotechnology, Life Sciences Center, Vilnius University, Sauletekio av. 7, LT-10257, Vilnius, Lithuania; Email: zare@ibt.It.

\section{References}

1. Song, J.-J., Smith, S. K., Hannon, G. J. \& Joshua-Tor, L. Crystal Structure of Argonaute and Its Implications for RISC Slicer Activity. Science (80-. ).305, 1434-1437 (2004).

2. Kwak, P. B. \& Tomari, Y. The N domain of Argonaute drives duplex unwinding during RISC assembly. Nat. Struct. Mol. Biol.19, 145-151 (2012).

3. Pratt, A. J. \& MacRae, I. J. The RNA-induced silencing complex: A versatile gene-silencing machine. J. Biol. Chem.284, 17897-17901 (2009).

4. Ryazansky, S., Kulbachinskiy, A. \& Aravin, A. A. The Expanded Universe of Prokaryotic Argonaute Proteins. MBio9, 1-20 (2018).

5. Swarts, D. C. et al. The evolutionary journey of Argonaute proteins. Nat. Struct. Mol. Biol.21, 743-753 (2014).

6. Lisitskaya, L., Aravin, A. A. \& Kulbachinskiy, A. DNA interference and beyond: structure and functions of prokaryotic Argonaute proteins. Nat. Commun.9, 1-12 (2018).

7. Kuzmenko, A. et al. DNA targeting and interference by a bacterial Argonaute nuclease. Nature (2020) doi:10.1038/s41586-020-2605-1.

8. Jolly, S. M. et al. Thermus thermophilus Argonaute Functions in the Completion of DNA Replication. Cel/182, 1545-1559.e18 (2020).

9. Boland, A., Huntzinger, E., Schmidt, S., Izaurralde, E. \& Weichenrieder, O. Crystal structure of the MIDPIWI lobe of a eukaryotic argonaute protein. Proc. Natl. Acad. Sci. U. S. A.108, 10466-10471 (2011).

10. Dayeh, D. M., Kruithoff, B. C. \& Nakanishi, K. Structural and functional analyses reveal the contributions of the $\mathrm{C}$ - and N-lobes of Argonaute protein to selectivity of RNA target cleavage. J. Biol. Chem.293, 6308-6325 (2018).

11. Frank, F., Hauver, J., Sonenberg, N. \& Nagar, B. Arabidopsis Argonaute MID domains use their nucleotide specificity loop to sort small RNAs. EMBO J.31, 3588-3595 (2012).

12. Frank, F., Sonenberg, N. \& Nagar, B. Structural basis for 5'-nucleotide base-specific recognition of guide RNA by human AG02. Nature465, 818-822 (2010). 
13. Till, S. et al. A conserved motif in Argonaute-interacting proteins mediates functional interactions through the Argonaute PIWI domain. Nat. Struct. Mol. Biol.14, 897-903 (2007).

14. Boland, A., Tritschler, F., Heimstädt, S., Izaurralde, E. \& Weichenrieder, O. Crystal structure and ligand binding of the MID domain of a eukaryotic Argonaute protein. EMBO Rep.11, 522-527 (2010).

15. Parker, J. S., Roe, S. M. \& Barford, D. Crystal structure of a PIWI protein suggests mechanisms for siRNA recognition and slicer activity. EMBO J.23, 4727-4737 (2004).

16. Ma, J. B. et al. Structural basis for $5^{\prime}$-end-specific recognition of guide RNA by the A. fulgidus Piwi protein. Nature434, 666-670 (2005).

17. Parker, J. S., Roe, S. M. \& Barford, D. Structural insights into mRNA recognition from a PIWI domainsiRNA guide complex. Nature434, 663-666 (2005).

18. Parker, J. S., Parizotto, E. A., Wang, M., Roe, S. M. \& Barford, D. Enhancement of the Seed-Target Recognition Step in RNA Silencing by a PIWI/MID Domain Protein. Mol. Cel/33, 204-214 (2009).

19. Blanchet, C. E. et al. Versatile sample environments and automation for biological solution X-ray scattering experiments at the P12 beamline (PETRA III, DESY). J. Appl. Crystallogr.48, 431-443 (2015).

20. Panjkovich, A. \& Svergun, D. I. CHROMIXS: Automatic and interactive analysis of chromatographycoupled small-angle X-ray scattering data. Bioinformatics34, 1944-1946 (2018).

21. Franke, D. \& Svergun, D. I. DAMMIF, a program for rapid ab-initio shape determination in small-angle scattering. J. Appl. Crystallogr.42, 342-346 (2009).

22. Svergun, D. I. Determination of the regularization parameter in indirect-transform methods using perceptual criteria. J. Appl. Crystallogr.25, 495-503 (1992).

23. Petoukhov, M. V. et al. New developments in the ATSAS program package for small-angle scattering data analysis. J. Appl. Crystallogr.45, 342-350 (2012).

24. Volkov, V. V. \& Svergun, D. I. Uniqueness of ab initio shape determination in small-angle scattering. J. Appl. Crystallogr.36, 860-864 (2003).

25. Svergun, D. I. Restoring low resolution structure of biological macromolecules from solution scattering using simulated annealing. Biophys. J.76, 2879-2886 (1999).

26. Svergun, D. I., Petoukhov, M. V. \& Koch, M. H. J. Determination of domain structure of proteins from $x-$ ray solution scattering. Biophys. J.80, 2946-2953 (2001).

27. Kozin, M. B. \& Svergun, D. I. Automated matching of high- and low-resolution structural models. J. Appl. Crystallogr.34, 33-41 (2001).

28. Tamulaitis, G. et al. Programmable RNA Shredding by the Type III-A CRISPR-Cas System of Streptococcus thermophilus. Mol. Cel/56, 506-517 (2014).

29. Svergun, D., Barberato, C. \& Koch, M. H. J. CRYSOL - a Program to Evaluate X-ray Solution Scattering of Biological Macromolecules from Atomic Coordinates. J. Appl. Crystallogr.28, 768-773 (1995).

30. Lyubchenko, Y. L., Shlyakhtenko, L. S. \& Ando, T. Imaging of nucleic acids with atomic force microscopy. Methods54, 274-283 (2011). 
31. Kapanidis, A. N. et al. Fluorescence-aided molecule sorting: Analysis of structure and interactions by alternating-laser excitation of single molecules. Proc. Natl. Acad. Sci. U. S. A.101, 8936-8941 (2004).

32. Tutkus, M., Sasnauskas, G. \& Rutkauskas, D. Probing the dynamics of restriction endonuclease NgoMIV-DNA interaction by single-molecule FRET. Biopolymers107, 1-9 (2017).

33. Cordes, T., Vogelsang, J. \& Tinnefeld, P. On the mechanism of trolox as antiblinking and antibleaching reagent. J. Am. Chem. Soc.131, 5018-5019 (2009).

34. Laskowski, R. A., Jabłonska, J., Pravda, L., Vareková, R. S. \& Thornton, J. M. PDBsum: Structural summaries of PDB entries. Protein Sci.27, 129-134 (2017).

35. Krissinel, E. \& Henrick, K. Inference of Macromolecular Assemblies from Crystalline State. J. Mol. Biol.372, 774-797 (2007).

36. Hegge, J. W. et al. DNA-guided DNA cleavage at moderate temperatures by Clostridium butyricum Argonaute. Nucleic Acids Res.47, 5809-5821 (2019).

37. Nicolai, C. \& Sachs, F. Solving ion channel kinetics with the QuB software. Biophys. Rev. Lett.8, 191211 (2013).

38. Lushnikov, A. Y., Potaman, V. N., Oussatcheva, E. A., Sinden, R. R. \& Lyubchenko, Y. L. DNA strand arrangement within the Sfil-DNA complex: Atomic force microscopy analysis. Biochemistry45, 152158 (2006).

39. Zaremba, M., Sasnauskas, G. \& Siksnys, V. The link between restriction endonuclease fidelity and oligomeric state: A study with Bse634I. FEBS Lett.586, 3324-3329 (2012).

40. Pathania, S., Jayaram, M. \& Harshey, R. M. Path of DNA within the Mu transpososome: Transposase interactions bridging two Mu ends and the enhancer trap five DNA supercoils. Cel/109, 425-436 (2002).

41. Peterson, G. \& Reznikoff, W. Tn5 transposase active site mutations suggest position of donor backbone DNA in synaptic complex. J. Biol. Chem.278, 1904-1909 (2003).

42. Hickman, A. B., Kailasan, S., Genzor, P., Haase, A. D. \& Dyda, F. Casposase structure and the mechanistic link between DNA transposition and spacer acquisition by CRISPR-Cas. Elife $9,1-26$ (2020).

43. Pouget, N., Turlan, C., Destainville, N., Salomé, L. \& Chandler, M. IS911 transpososome assembly as analysed by tethered particle motion. Nucleic Acids Res.34, 4313-4323 (2006).

44. Bellamy, S. R. W., Mina, P., Retter, S. E. \& Halford, S. E. Fidelity of DNA Sequence Recognition by the Sfil Restriction Endonuclease Is Determined by Communications between Its Two DNA-Binding Sites. J. Mol. Biol.384, 557-563 (2008).

45. Swarts, D. C. et al. DNA-guided DNA interference by a prokaryotic Argonaute. Nature507, 258-261 (2014).

46. Olovnikov, I., Chan, K., Sachidanandam, R., Newman, D. K. \& Aravin, A. A. Bacterial Argonaute Samples the Transcriptome to Identify Foreign DNA. Mol. Cel/51, 594-605 (2013). 
47. Wright, A. V et al. Structures of the CRISPR genome integration complex. Science (80-. ).357, 1113 LP - 1118 (2017).

48. Xiao, Y., Ng, S., Hyun Nam, K. \& Ke, A. How type II CRISPR-Cas establish immunity through Cas1Cas2-mediated spacer integration. Nature550, 137-141 (2017).

49. Hare, S., Gupta, S. S., Valkov, E., Engelman, A. \& Cherepanov, P. Retroviral intasome assembly and inhibition of DNA strand transfer. Nature464, 232-236 (2010).

50. Grawenhoff, J. \& Engelman, A. N. Retroviral integrase protein and intasome nucleoprotein complex structures. World J. Biol. Chem.8, 32 (2017).

51. Fu, L. et al. The prokaryotic Argonaute proteins enhance homology sequence-directed recombination in bacteria. Nucleic Acids Res.47, 3568-3579 (2019).

52. Ingargiola, A., Lerner, E., Chung, S. Y., Weiss, S. \& Michalet, X. FRETBursts: An open source toolkit for analysis of freely-diffusing Single-molecule FRET. PLoS One11, 1-27 (2016).

53. Holden, S. J. et al. Defining the Limits of Single-Molecule FRET Resolution in TIRF Microscopy. Biophys. J.99, 3102-3111 (2010).

54. Durand, D. et al. NADPH oxidase activator p67phox behaves in solution as a multidomain protein with semi-flexible linkers. J. Struct. Biol.169, 45-53 (2010).

55. Chandran, S. Rapid Assembly of DNA via Ligase Cycling Reaction (LCR). in Synthetic DNA: Methods and Protocols (ed. Hughes, R. A.) 105-110 (Springer New York, 2017). doi:10.1007/978-1-4939-63430_8.

56. Konarev, P. V., Volkov, V. V., Sokolova, A. V., Koch, M. H. J. \& Svergun, D. I. PRIMUS - a Windows-PC based system for small-angle scattering data analysis. J. Appl. Crystallogr.36, 1277-1282 (2003).

57. Fischer, H., De Oliveira Neto, M., Napolitano, H. B., Polikarpov, I. \& Craievich, A. F. Determination of the molecular weight of proteins in solution from a single small-angle X-ray scattering measurement on a relative scale. J. Appl. Crystallogr.43, 101-109 (2010).

\section{Tables}

Table 1. AfAgo-DNA complexes observed by AFM.

\begin{tabular}{|lllll|}
\hline Protein & $\begin{array}{l}\text { DNA loops, } \\
\%\end{array}$ & $\begin{array}{l}\text { Linear } \\
\begin{array}{l}\text { Bound to one } \\
\text { end, \% }\end{array}\end{array}$ & $\begin{array}{l}\text { Bound to both } \\
\text { ends, \% }\end{array}$ & $\begin{array}{l}\text { Total number of analyzed } \\
\text { complexes }\end{array}$ \\
\hline $\begin{array}{l}\text { WT } \\
\text { AfAgo }\end{array}$ & 55 & 29 & 16 & 208 \\
\hline AfAgo $\Delta$ & 12 & 29 & 59 & 319 \\
\hline
\end{tabular}




\section{Figures}

a

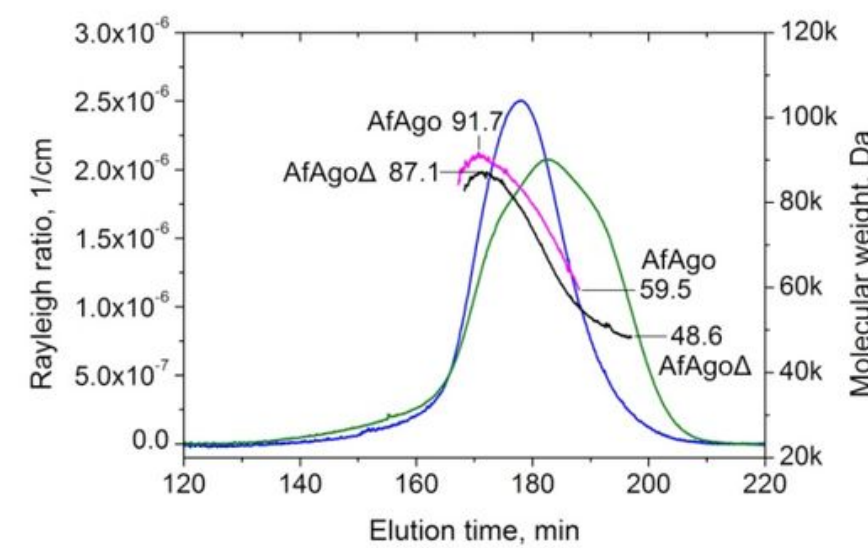

b

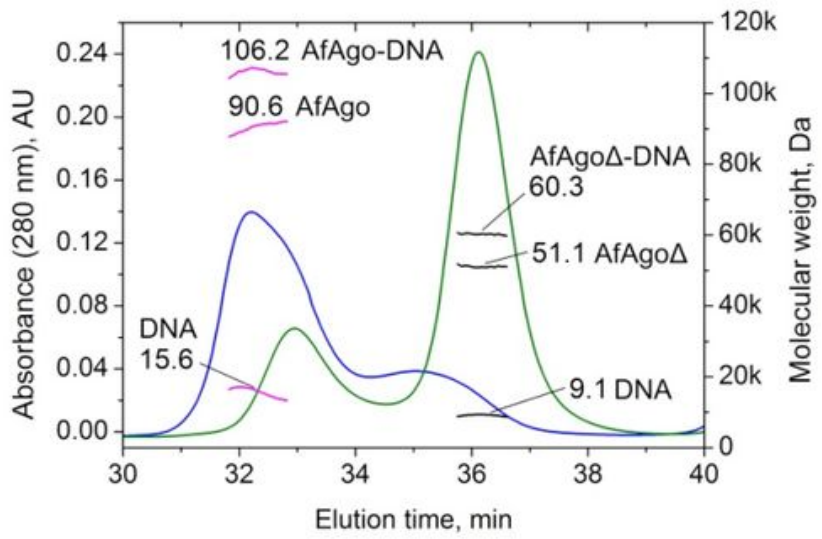

AfAgo
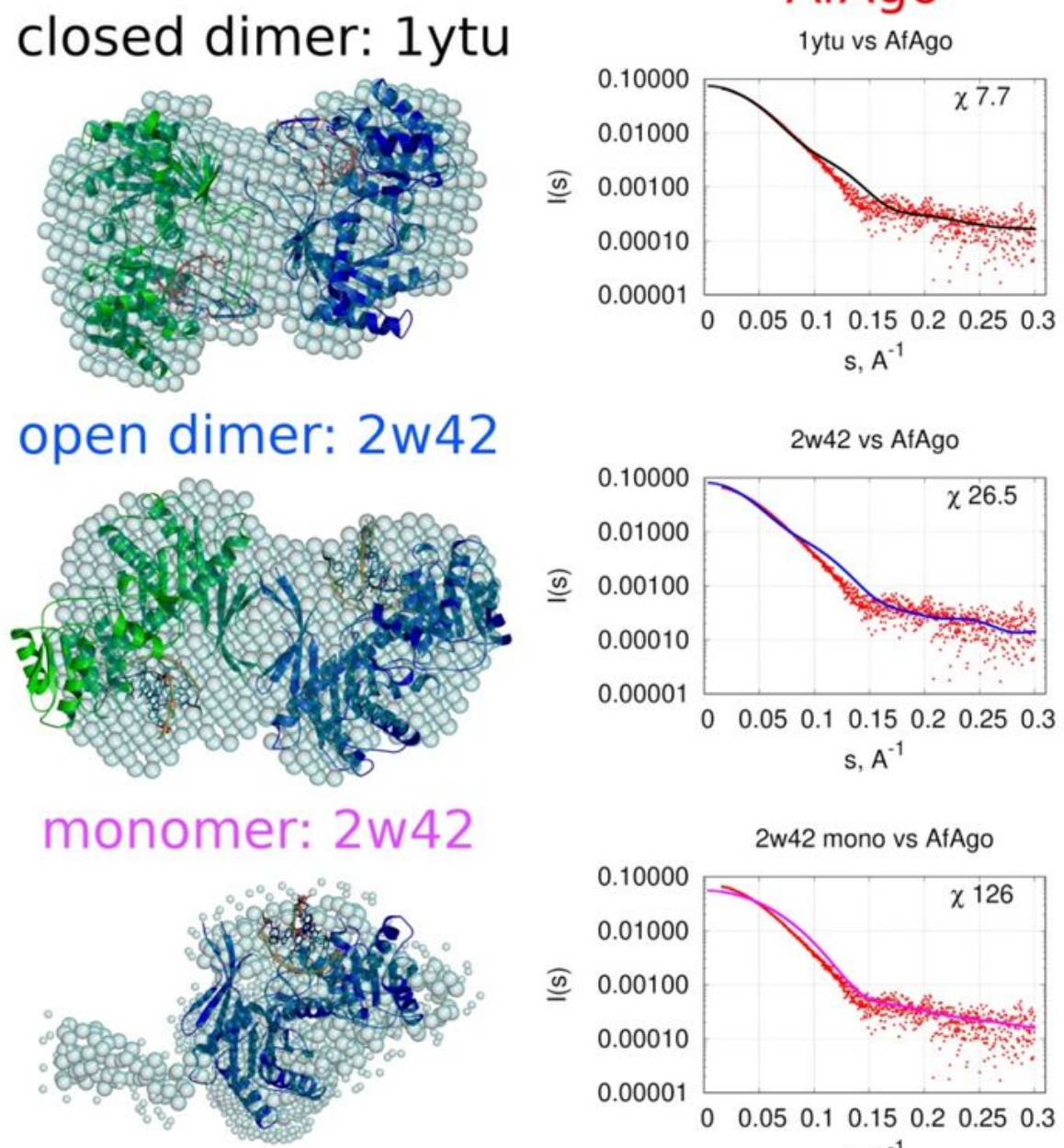
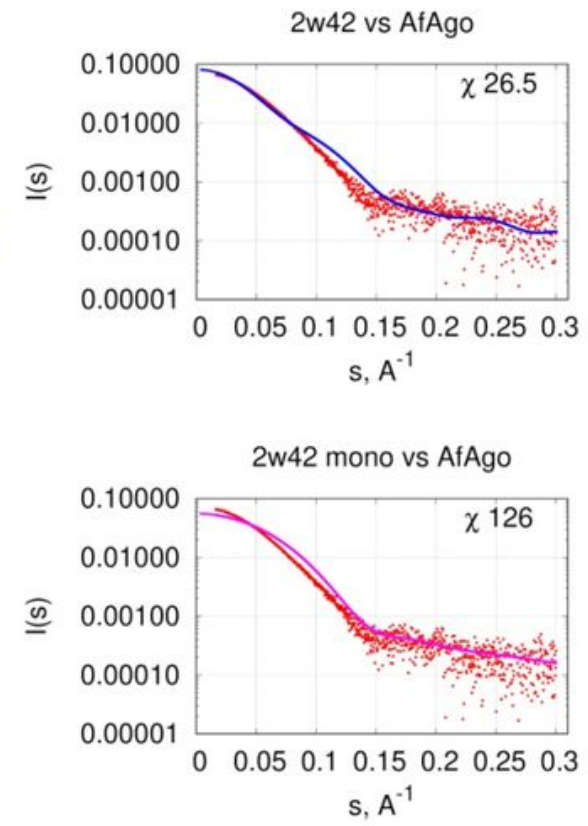

AfAgo $\triangle$

1ytu vs AfAgo $\Delta$

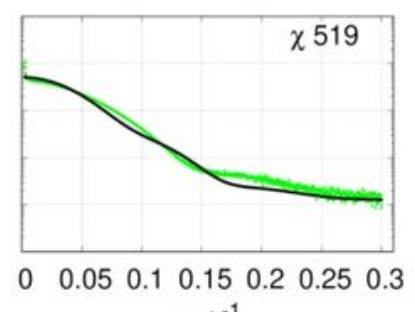

$\mathrm{s}, \mathrm{A}^{-1}$

$2 w 42$ vs AfAgo $\Delta$

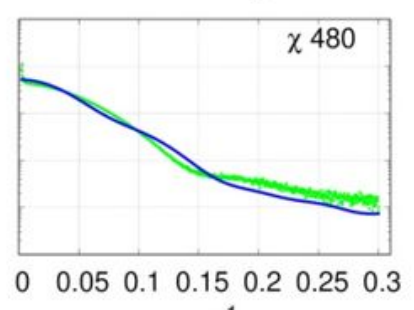

s, $A^{-1}$

$2 w 42$ vs AfAgo $\Delta$

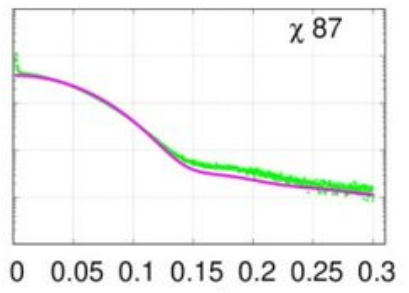

s, $A^{-1}$

Figure 1

SEC-MALS and SAXS analysis of apo-AfAgo and AfAgo-DNA complexes. (a) SEC-MALS analysis of WT AfAgo and dimerization mutant AfAgo $\triangle$ unbound to nucleic acids. The light scattering data (blue for WT AfAgo, green for $A f A g o \Delta$ ) is shown along with the calculated Mw values (magenta for WT, black for 
mutant). The highest and lowest $\mathrm{Mw}$ values calculated for each protein are indicated. Theoretical Mw of WT AfAgo monomer is $50.8 \mathrm{kDa}$, theoretical Mw of AfAgo $\triangle$ monomer is $49.9 \mathrm{kDa}$. (b) SEC-MALS analysis of AfAgo-DNA and AfAgo $\triangle$-DNA complexes. The UV absorption data of AfAgo-DNA (blue) and AfAgo $\triangle$ DNA (green) is shown along the Mw values of full complexes, the protein component and the DNA component (magenta for AfAgo-DNA sample and black for AfAgo $\triangle$-DNA sample, respectively). The theoretical Mw of a 2:2 WT AfAgo:DNA complex is $119 \mathrm{kDa}(2 \times 50.8+2 \times 8.7 \mathrm{kDa})$, theoretical Mw of a 1:1 AfAgo $\triangle$-DNA complex is $58.6 \mathrm{kDa}(49.9+8.7 \mathrm{kDa})$. (c) SAXS data of WT AfAgo complex with MZ-1289 DNA (red points), and the dimerization mutant AfAgo $\triangle$ with MZ-1289 DNA (green points) are compared with the scattering curves generated from the "closed" dimer with dsRNA (PDB ID: 1ytu, black curves), "open" dimer (PDB ID: 2w42, blue curves) and AfAgo-DNA complex (PDB ID: 2w42, magenta curves) by CRYSOL. Corresponding AfAgo structures are shown in the second column superimposed with the dummy atom models generated using the SAXS data of AfAgo complex with MZ-1289 oligoduplex.

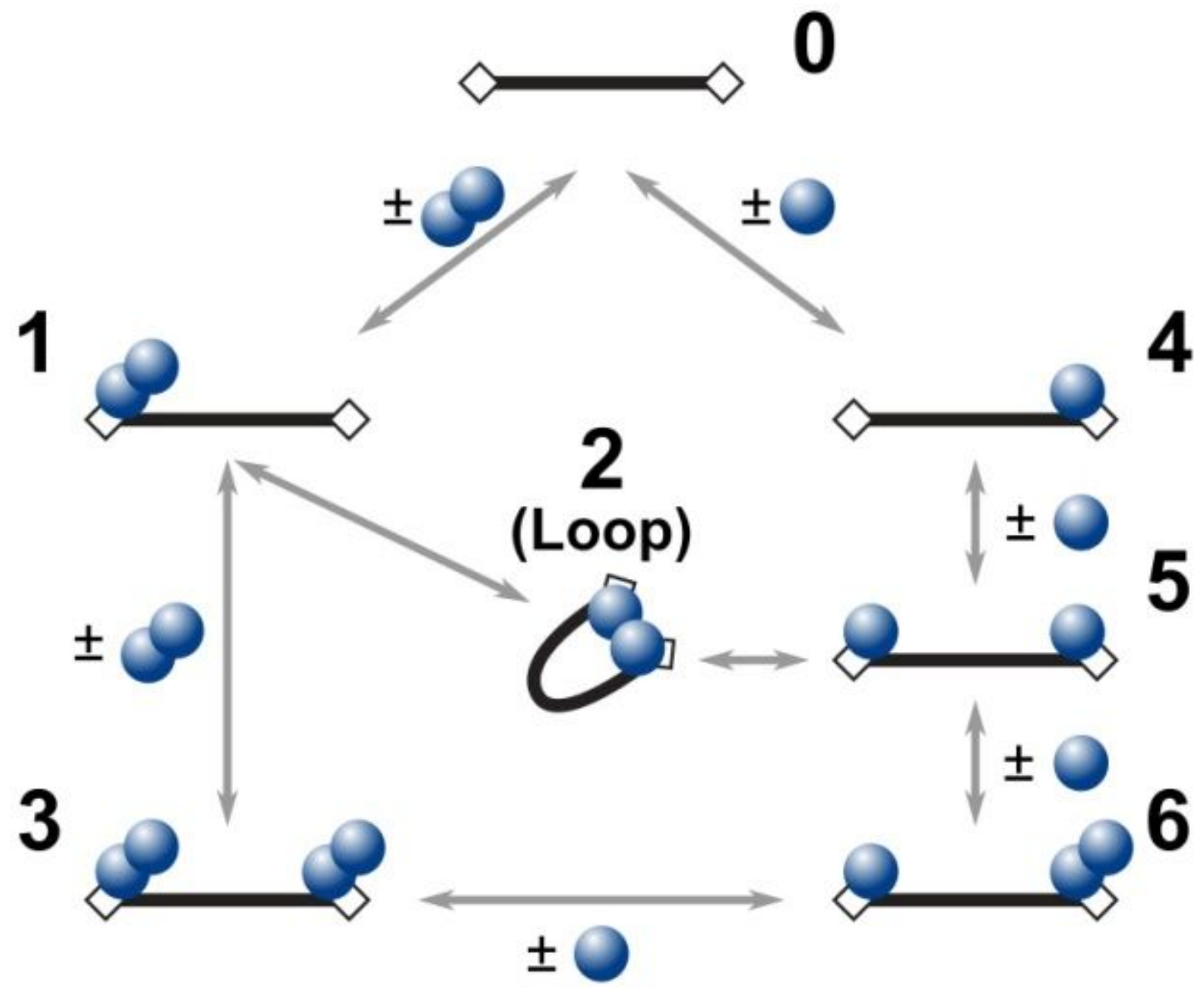

Figure 1

Kinetic schemes depicting possible reaction pathways between two-target site DNA fragment and WT AfAgo. Black bars represent DNA, rectangular boxes - AfAgo-binding targets (DNA ends), blue circles AfAgo monomers. Note that species 1 and 6 may be formed via alternative pathways not depicted in the 
scheme (species 1 may be formed when two monomers associate consecutively with the same DNA end; species 6 may be formed when a monomer and a dimer associated with different DNA ends).

a

$$
\text { Zero-FRET } \quad \text { FRET }
$$

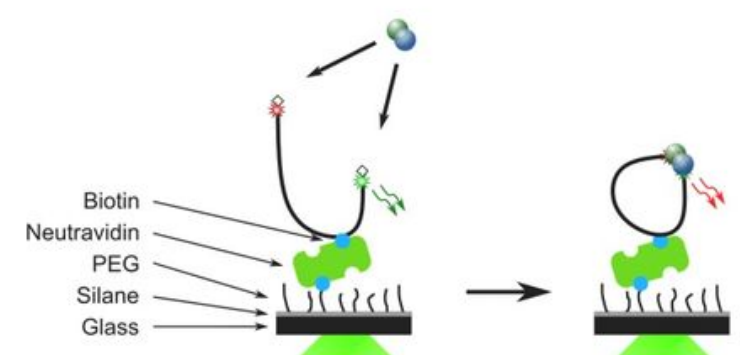

b

\section{C}
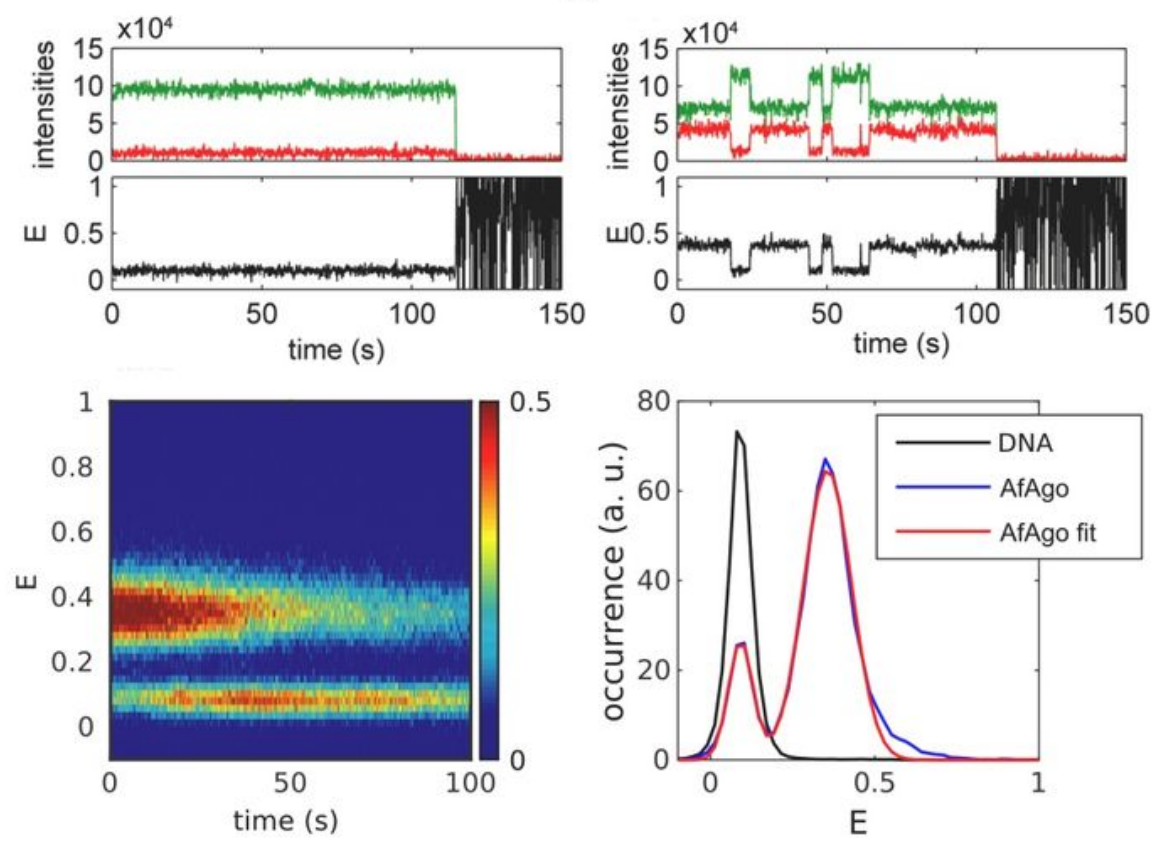

e
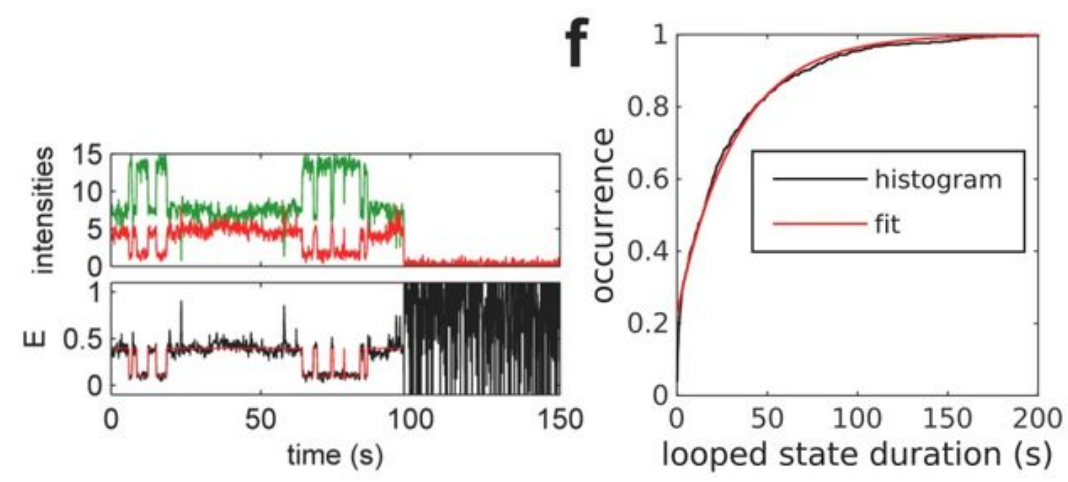

\section{Figure 1}

Dynamics of WT AfAgo-induced DNA loops. (a) A schematic overview of the single-molecule assay using TIRF microscopy. (b, c) Trajectories of donor (green) and acceptor (red) intensity and corresponding proximity ratio, E, of individual DNA fragments without (b) and with $10 \mathrm{nM}$ AfAgo (c). (d) Left - an image 
of 287 pooled time traces of the proximity ratio, E, from the measurement with $10 \mathrm{nM}$ of AfAgo. The image is normalized to the maximum image intensity. Right - a section of the image in the left integrated over the first $10 \mathrm{~s}$ shown with the two-Gaussian fit. The positions of the Gaussian maxima are $0.09 \pm 0.01$ and $0.36 \pm 0.01$. For comparison a trace- and time-averaged section from the measurement of 227 traces on bare DNA is shown. (e) An example of trajectories of donor (green) and acceptor (red) intensity and corresponding proximity ratio, E, with HMM idealization of an individual DNA fragment with $10 \mathrm{nM}$ AfAgo. (f) - cumulative histogram of the looped state durations from $287 \mathrm{E}$ traces with a singleexponential fit with the exponential factor of $33 \pm 1 \mathrm{~s}$.

a

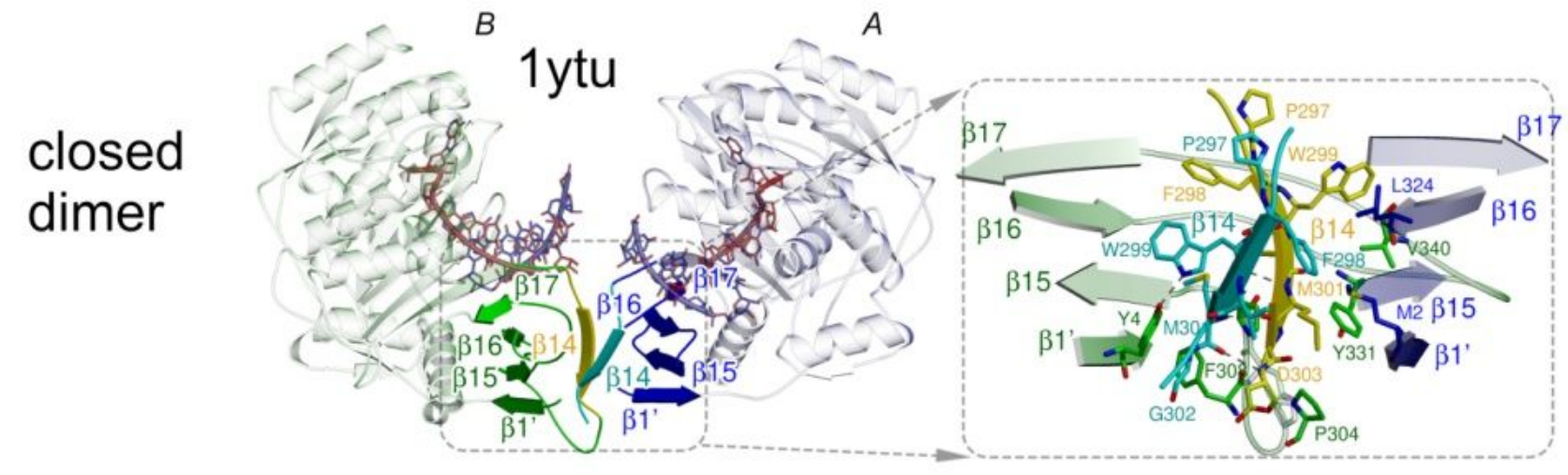

b

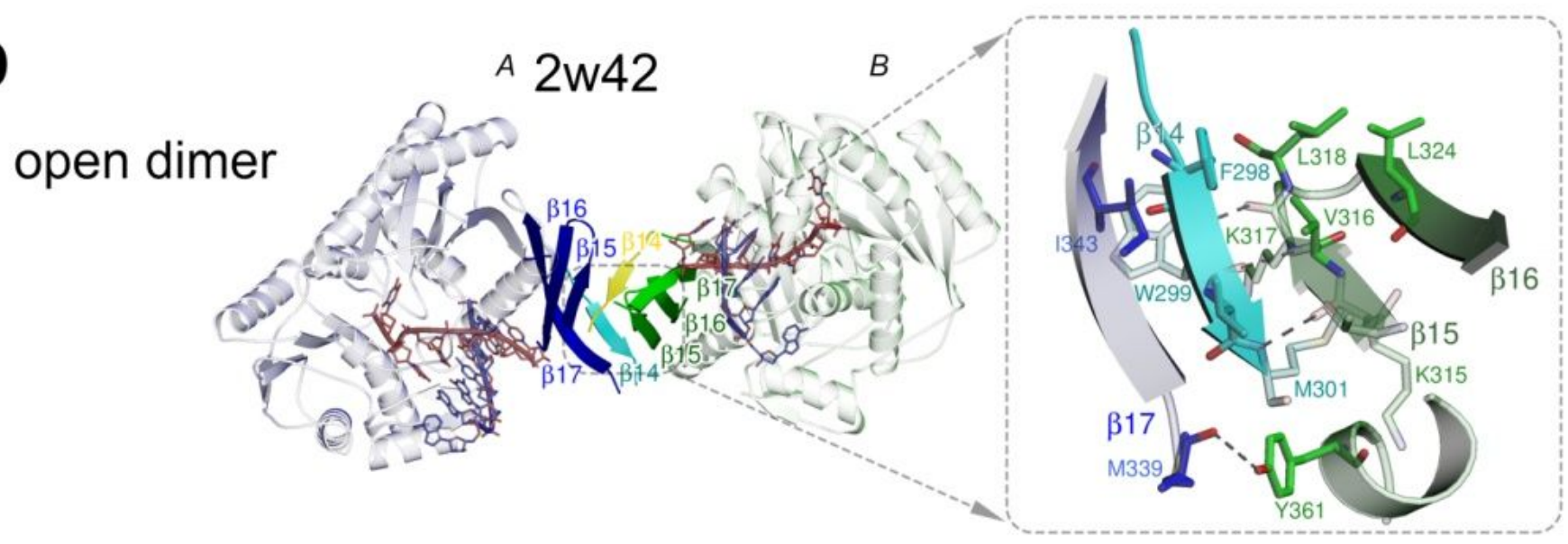

\section{Figure 1}

Dimerization of AfAgo. (a-b). Protein subunits are colored blue (protein chain A) and green (protein chain $B)$. The interface-forming secondary structure elements are highlighted and numbered according to the PDB ID 2w42 assignment made by PDBsum [34]. The "guide" DNA/RNA strands bound by AfAgo are colored red, "target" strands - blue. Residues 296-303 deleted in AfAgo $\triangle$ are colored cyan and yellow. Hydrogen bonds are shown as dashed lines. (a) AfAgo complex with dsRNA (PDB ID 1ytu, both protein chains as present in ASU), the "closed" dimer [16]. (b), AfAgo complex with dsDNA (PDB ID 2w42) [18]) the "open" dimer. $\beta$-strands from both subunits assemble into a closed $\beta$-barrel structure, with intersubunit interface formed by $\beta 14$ and $\beta 15$ strands of neighboring subunits. 


\section{WT AfAgo}
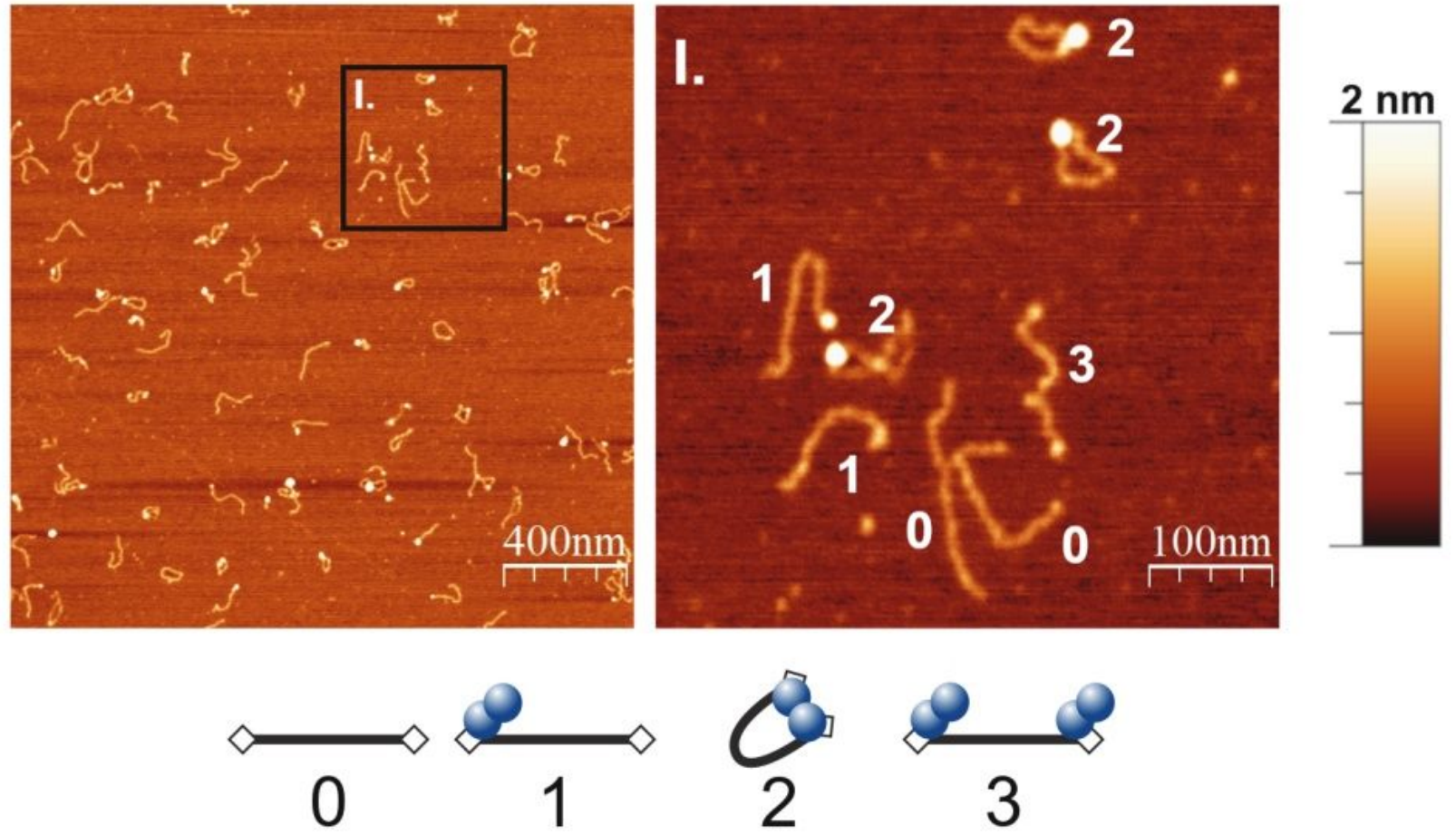

\section{AfAgo $\Delta$}
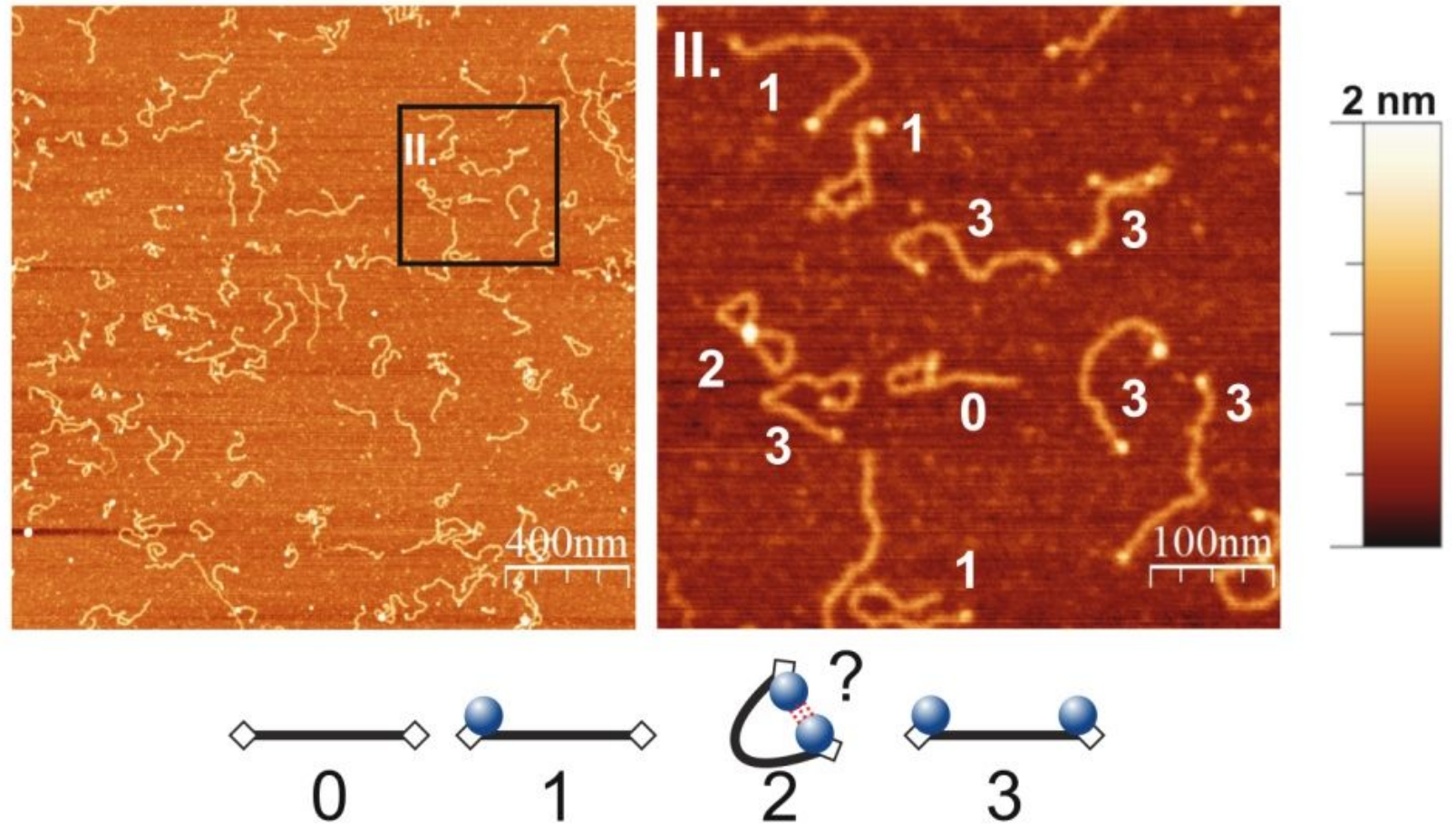

\section{Figure 1}

Visualization of AfAgo-induced DNA loops by AFM. Representative AFM images and enlarged views of DNA-protein complexes adsorbed to APS-mica acquired in the air are shown. WT AfAgo-DNA (top) and AfAgo $\Delta$-DNA (bottom), area of each left column image is $4 \mu \mathrm{m} 2$, scale bar is $400 \mathrm{~nm}$. Right column shows regions marked by squares in the respective images on the left, enlarged 4 -fold, scale bar $100 \mathrm{~nm}$. Various observed species are depicted below the respective images and are marked as follows: "0" - 
naked DNA; "1" - protein bound to one DNA end; "3" - protein bound to both DNA ends; "2" - ring-shaped (looped) DNA. In the case of AfAgo $\triangle$, species " 2 " is presumably formed due to glutaraldehyde crosslinking. The $Z$ scale bar is $2 \mathrm{~nm}$.

a

Zero-FRET

FRET

AfAgo
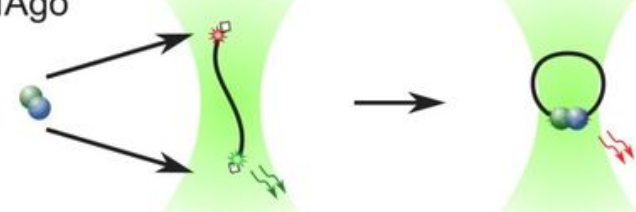

b

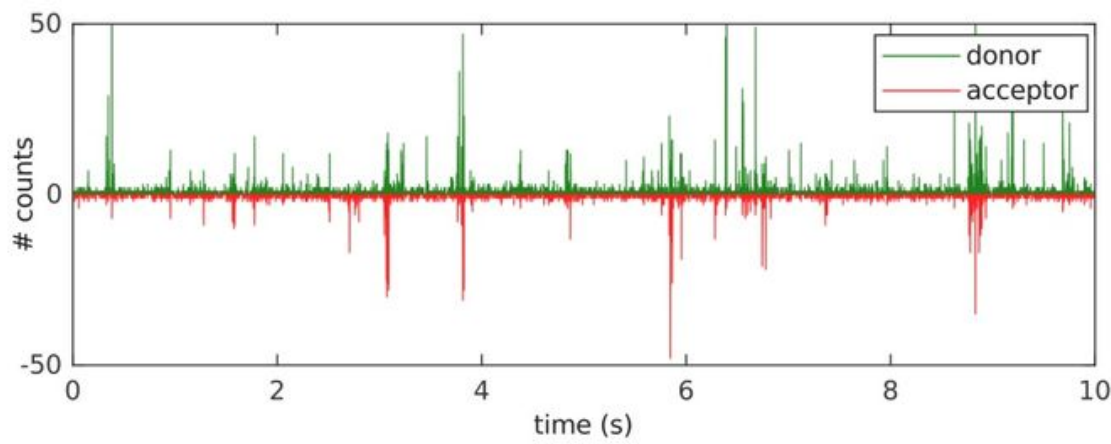

C

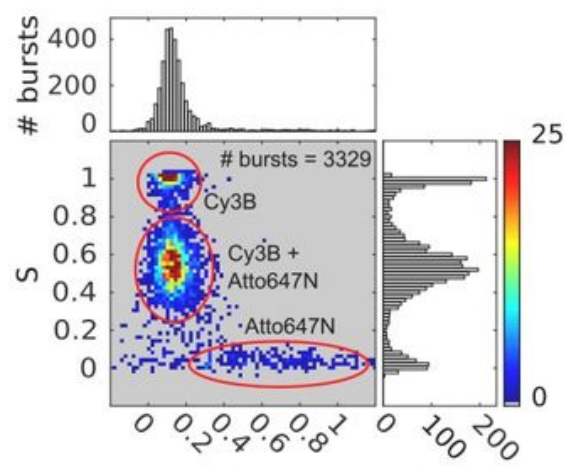

d

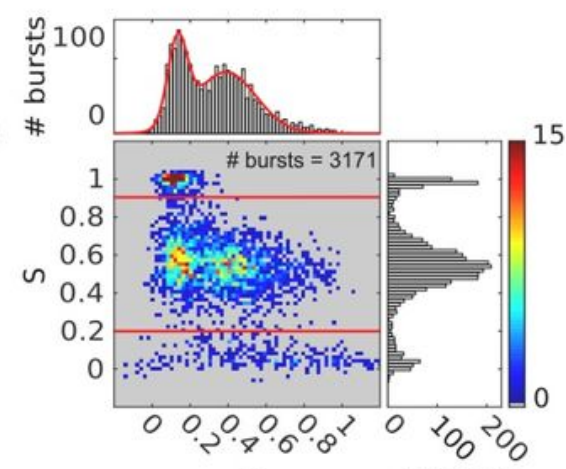

$\mathrm{E}$

\# bursts
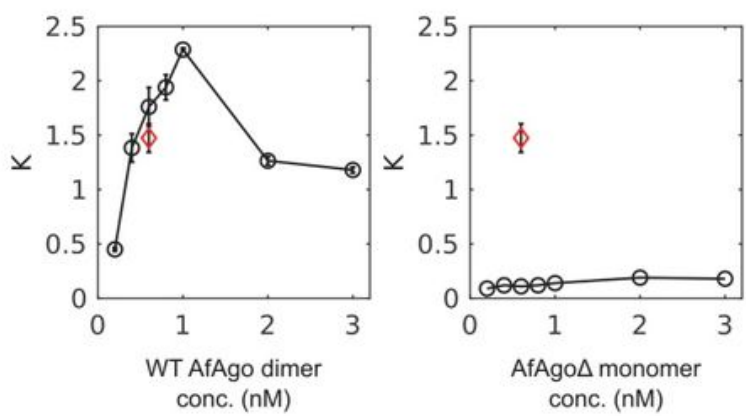

Figure 1

Single-molecule studies of AfAgo-DNA interactions in solution. (a) A schematic overview of the single molecule assay. Left, free DNA; right, WT AfAgo-DNA (blue and green circles) complex in a looped state. 
(b) Fluorescence intensity trace with 1 ms time bin of 25 pM DNA with 1 nM AfAgo. Red: inverted acceptor fluorescence upon donor excitation, green: donor fluorescence upon donor excitation. (c) Left - E$S$ histogram of DNA alone. The top and side axes contain, respectively, one-dimensional E (proximity ratio) and $S$ (donor/acceptor stoichiometry) histograms of all bursts. Denoted are areas representing donor-only DNA, acceptor-only DNA, and dual-labeled DNA. Right - E-S histogram of DNA with $1 \mathrm{nM}$ AfAgo. The one-dimensional $E$ histogram on top is derived from bursts with $S=0.2-0.9$, designated by horizontal lines in the E-S histogram. The red curve is a two-Gaussian fit to the data that gave the positions of the Gaussian maxima on the E-axis $(0.13 \pm 0.01$ and $0.39 \pm 0.02)$. (d) Left - dependence of the ratio of looped and unlooped DNA molecules (parameter K) on WT AfAgo concentration (open circles). Right - the dependence of $\mathrm{K}$ on the AfAgo $\triangle$ concentration (open circles). The red diamonds in both graphs represent the competition experiment performed with $0.6 \mathrm{nM}$ WT AfAgo dimer and $0.6 \mathrm{nM}$ AfAgo $\Delta$ monomer. All data points are average values of three measurements \pm 1 standard deviation.

\section{Supplementary Files}

This is a list of supplementary files associated with this preprint. Click to download.

- SupportinginfoGolovinasetal2020manuscriptSciRep.pdf 